\title{
Absence of Orthogonality Catastrophe after a Spatially Inhomogeneous Interaction Quench in Luttinger Liquids
}

\author{
Balázs Dóra ${ }^{1, *}$ and Frank Pollmann ${ }^{2}$ \\ ${ }^{1}$ Department of Physics and BME-MTA Exotic Quantum Phases Research Group, \\ Budapest University of Technology and Economics, 1521 Budapest, Hungary \\ ${ }^{2}$ Max-Planck-Institut für Physik komplexer Systeme, 01187 Dresden, Germany
}

(Received 30 October 2014; published 27 August 2015)

\begin{abstract}
We investigate the Loschmidt echo, the overlap of the initial and final wave functions of Luttinger liquids after a spatially inhomogeneous interaction quench. In studying the Luttinger model, we obtain an analytic solution of the bosonic Bogoliubov-de Gennes equations after quenching the interactions within a finite spatial region. As opposed to the power-law temporal decay following a potential quench, the interaction quench in the Luttinger model leads to a finite, hardly time-dependent overlap; therefore, no orthogonality catastrophe occurs. The steady state value of the Loschmidt echo after a sudden inhomogeneous quench is the square of the respective adiabatic overlaps. Our results are checked and validated numerically on the $X X Z$ Heisenberg chain.
\end{abstract}

DOI: 10.1103/PhysRevLett.115.096403

PACS numbers: 71.10.Pm, 05.70.Ln, 67.85.-d

Introduction.-The sensitivity of a quantum time evolution to perturbations is a central problem in many distinct areas of physics. Starting probably from a discussion between Loschmidt and Boltzmann, the effect of time forward and reversed processes has always enjoyed the attention of prominent researchers. The revival of interest towards nonequilibrium time evolution has been triggered by recent experiments in cold atomic gases, and questions such as thermalization and equilibration [1], defect production during passage through a quantum critical point $[2,3]$, quantum work fluctuation relations [4], etc. call for developments from both the experimental and theoretical sides.

Nonequilibrium states can be reached in many different ways. While the condensed matter thinking typically implies the application of strong electric and magnetic fields [5], cold atomic systems offer the possibility to change interactions by tuning to or away from a Feshbach resonance or by altering the lattice parameters [6]. From the latter class, quantum quenching the interaction strength has been studied in a variety of systems [2,3]. The common feature in these approaches is an abrupt change of a spatially homogeneous interaction parameter. However, the consideration of spatially inhomogeneous quenches can be equally exciting [7-10]. The famous example is the $\mathrm{x}$-ray edge problem [11], where a spatially local (therefore highly inhomogeneous in $k$ space) potential scatterer is switched on abruptly, realizing the time-dependent version of Anderson's orthogonality catastrophe (OC).

Inspired by these, we focus on the interplay of spatial inhomogeneity and strong correlations on the nonequilibrium dynamics by studying an inhomogeneous interaction quench in a Luttinger liquid (LL). The prototypical dynamical quantity, central to $\mathrm{x}$-ray edge physics, is the Loschmidt echo (LE) [12], which is the overlap of two wave functions, $\left|\Psi_{0}(t)\right\rangle$ and $|\Psi(t)\rangle$, evolved from the same initial state but with different Hamiltonians, $H_{0}$ and $H$,

$$
\mathcal{L}(t) \equiv\left|\left\langle\Psi_{0}(t) \mid \Psi(t)\right\rangle\right|^{2}
$$

Inhomogeneous quantum quenches have been well studied in various systems [11,13-17]. For relevant perturbations, the LE decays in a power-law fashion with a universal exponent, while for a marginal defect, the exponent depends on the final strength of the perturbation [15]. After an inhomogeneous quench over a finite spatial region $l$, excitations are mostly produced within the quenched region. A potential quench of strength $h$ within a region $l$ keeps the LE close to 1 for times $t \ll l / \mathrm{v}_{F}\left(\mathrm{v}_{F}\right.$ is the maximal propagation velocity) as the quasiparticles in the quenched region feel a chemical potential shift, which does not destroy the coherence. For long times $t \gg l / \mathrm{v}_{F}$, the created excitations have left the quenched region, and the overlap decays in a power-law fashion. The exponent is determined by the phase shift $\sim\left(l h / \mathrm{v}_{F}\right)^{2}$ without additional interactions, when the potential is marginal. In the presence of repulsive interactions, i.e., in a Luttinger liquid, a local potential is a relevant perturbation, yielding a universal decay exponent $[13,15]$.

For an inhomogeneous interaction quench, the focus of this work, the LE in the short time regime is expected to scale similarly to that after a homogeneous quench, after replacing the system size by $l$. The long time response of the system is, however, not obvious at all. Based on the $\mathrm{x}$ ray edge problem, one would expect the LE to vanish with $t$, since local backscattering terms are inevitably induced at the boundary of the interacting region [18]. As opposed to this, we show that no OC occurs for an inhomogeneous interaction quench in one spatial dimension and the LE 
in the $l \gtrless v_{F} t$ regions remains finite. Our analytical calculations are checked and confirmed numerically using time-dependent density matrix renormalization group (tDMRG-)based methods [19-22] on the $X X Z$ Heisenberg model, which contains local backscattering terms at the boundary of the interacting region.

Our work is interesting and relevant not only for its condensed matter and cold atomic aspect, but it bears importance in nuclear physics as well, where the overlap of (fermionic) Hartree-Fock-Bogoliubov wave functions is essential in determining the properties of nuclear states $[23,24]$ as it accounts for deformation and pairing.

Inhomogeneous Luttinger liquids.-We investigate the spatially inhomogeneous and time-dependent $X X Z$ Heisenberg model, which reads as

$H=\sum_{n=-L / 2}^{L / 2-1} J\left(S_{n}^{x} S_{n+1}^{x}+S_{n}^{y} S_{n+1}^{y}\right)+J_{z}(t) \sum_{n=-l / 2}^{l / 2-1} S_{n}^{z} S_{n+1}^{z}$,

where $n$ indexes the lattice sites and $J>0$ is the antiferromagnetic exchange interaction. We are going to manipulate $J_{z}<J$ via a sudden quench as $J_{z}(t)=$ $J_{z} \Theta(t)$, with $\Theta(t)$ the Heaviside function. The spatial width of the interaction is $l$, and smoothing the envelope function does not alter our results. The effective low-energy dynamics of the Heisenberg model is described by an "elastic string" [11,25], whose first quantized form is

$$
H_{0}=\frac{\mathbf{v}_{F}}{2 \pi} \int_{-L / 2}^{L / 2} d x\left\{[\pi \Pi(x)]^{2}+\left[\partial_{x} \phi(x)\right]^{2}\right\},
$$

where $\psi$ and $\Pi$ are conjugate variables with $[\phi(x), \Pi(y)]=$ $i \delta(y-x)$ and $L$ is the length of the system. The inhomogeneous interaction quench of the Heisenberg model induces

$$
H_{\text {int }}(t>0)=\frac{g K_{0}}{2 \pi} \int_{-l / 2}^{l / 2} d x\left[\partial_{x} \phi(x)\right]^{2},
$$

where $K_{0}$ emanates from a homogeneous interaction in the initial state and $l=L$ represents a global quench. The LL parameter in the quenched region is $K=$ $K_{0} / \sqrt{1+\left(g K_{0} / \mathrm{v}_{F}\right)}$, while the renormalized velocity is $\mathrm{v}=\mathrm{v}_{F} K_{0} / K$. For the Heisenberg model, the LL parameter is $K=\pi / 2\left[\pi-\arccos \left(J_{z} / J\right)\right.$ ] [25]. The effective Gauss model is sketched in Fig. 1. The equilibrium and transport properties of the spatially inhomogeneous LLs have long been investigated [26-29]. Note that a spatially abrupt interaction barrier in Eq. (2) also induces backscattering [18], neglected in the above continuum description. Surprisingly, the LE is not affected by these terms, as demonstrated below by a careful comparison between bosonization and tDMRG.

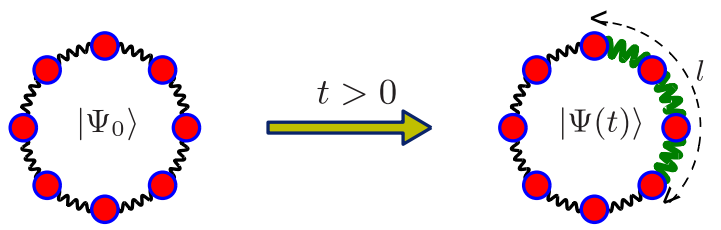

FIG. 1 (color online). The low-energy dynamics of the $X X Z$ Heisenberg chain is faithfully represented by the Luttinger model. An inhomogeneous interaction quench in the Luttinger model is equivalent to the continuum limit of a harmonic chain, sketched in the figure, where the spring constants (and/or masses) of a region of size $l$ are altered abruptly at $t=0$.

The time evolved wave function $\Psi(t)=U(t) \Psi_{0}$, where the time evolution is governed by Eq. (4) as

$$
U(t)=\mathcal{T} \exp \left(-i \int_{0}^{t} H_{\text {int }}\left(t^{\prime}\right) d t^{\prime}\right) .
$$

This time evolution operator can be calculated using a linked cluster or cumulant expansion techniques [11,30], similarly to how the x-ray edge problem was approached. However, for the present problem, it is more advantageous to generalize the results of the homogeneous system [31,32] for the inhomogeneous case.

The total Hamiltonian is rewritten as

$$
H(t>0)=\Phi^{\dagger}\left(\begin{array}{ll}
\Omega & G \\
G & \Omega
\end{array}\right) \Phi
$$

where $\Phi^{\dagger}=\left(b_{k_{1}}^{\dagger}, b_{k_{2}}^{\dagger}, \ldots, b_{k_{N}}^{\dagger}, b_{k_{1}}, b_{k_{2}}, \ldots, b_{k_{N}}\right)$ and $k_{1, \ldots, N}$ are integer multiples of $2 \pi / L$, excluding $k=0$. The symmetric blocks are defined as

$$
\begin{gathered}
\Omega(k, p)=\mathrm{v}_{F}|k| \delta_{k, p}+\frac{g K_{0}}{2 L} \sqrt{|k p|} \tilde{f}(k-p, l), \\
G(k, p)=\frac{g K_{0}}{2 L} \sqrt{|k p|} \tilde{f}(k+p, l),
\end{gathered}
$$

and $\tilde{f}(k, l)$ is the Fourier transform of the envelope function of the interaction, which is $\tilde{f}(k, l)=2 \sin (k l / 2) / k$ for an abruptly terminated interaction over $l$ [see Eq. (2)]. For $t<0, g=0$. The matrix structure of $\Omega$ and $G$ naturally favors a sharp momentum cutoff $q_{c}$. The time evolution of the bosons is determined from the Heisenberg equation of motion, whose solution is written in the concise form as $\Phi(t)=U(t) \Phi(0)$, where the time evolution operator defines the generalized Bogoliubov matrices, $u(t)$ and $v(t)$, similarly to the homogeneous case [31], as

$$
U(t)=\exp \left[-i t\left(\begin{array}{cc}
\Omega & G \\
-G & -\Omega
\end{array}\right)\right] \equiv\left(\begin{array}{cc}
u & v \\
v^{*} & u^{*}
\end{array}\right),
$$

and the LE is expressed in terms of the regularized Fredholm determinant [33] 


$$
\mathcal{L}(t)=1 / \sqrt{\operatorname{det}\left(1+v^{\dagger} v\right)},
$$

and $v^{\dagger}=-v$. The fermionic version of Eq. (10) in equilibrium is known as the Onishi formula [24].

Using Ref. [34], the generalized Bogoliubov coefficients are expressed in terms of the Hamiltonian matrix blocks as

$$
\begin{aligned}
v= & \frac{\cos (t \tilde{\omega})-\cos (t \tilde{\omega})^{T}}{2}+i \frac{\Omega}{2}\left[\frac{\sin (t \tilde{\omega})}{\tilde{\omega}}-\left(\frac{\sin (t \tilde{\omega})}{\tilde{\omega}}\right)^{T}\right] \\
& +i \frac{G}{2}\left[\frac{\sin (t \tilde{\omega})}{\tilde{\omega}}+\left(\frac{\sin (t \tilde{\omega})}{\tilde{\omega}}\right)^{T}\right],
\end{aligned}
$$

where $\tilde{\omega}=\sqrt{(\Omega-G)(\Omega+G)}$, and $u$ is obtained similarly, though it is not needed for our purposes. The commutator $\left[\tilde{\omega}, \tilde{\omega}^{T}\right] \neq 0$ for an inhomogeneous quench, and vanishes only for a homogeneous quench, when only the last term is present in $v$. With Eqs. (10) and (11), the asymptotic long time limit of the overlap (taken after the thermodynamic limit is taken) is given by

$$
\begin{aligned}
\mathcal{L}_{\infty} & \equiv \mathcal{L}(t \rightarrow \infty) \\
& =\operatorname{det}\left[\frac{1}{2}+\frac{\Omega}{4}\left(\tilde{\omega}^{-1}+\tilde{\omega}^{-1 T}\right)+\frac{G}{4}\left(\tilde{\omega}^{-1}-\tilde{\omega}^{-1 T}\right)\right]^{-1} .
\end{aligned}
$$

The long time limit of the sudden quench overlap is the square of the overlap of the adiabatic ground state wave functions, $\mathcal{L}_{\infty}=\mathcal{L}_{\text {g.s. }}^{2}$. This extends the previous results in Ref. [31] to the more general inhomogeneous quench case. A finite value of $\mathcal{L}_{\infty}$ would indicate the absence of an OC for an inhomogeneous interaction quench. The heart of the evaluation is the $v^{\dagger} v$ matrix, which is diagonal in the homogeneous case, yielding the result of Ref. [31]. In the following, we will analyze various special cases of Eq. (12).

Local quench region $\left(q_{c} l \ll 1\right)$. - This limit is obtained by taking $l \rightarrow 0$ but keeping $g l$ fixed; therefore, $\tilde{f}(k, l)=l$, and the LE depends only on the dimensionless number $K_{0} g l q_{c} / \mathrm{v}_{F}$. This local interaction quench limit does not apply directly to LLs because many additional local terms, neglected in the Gauss model, can play an important role, as is the case for the x-ray edge problem [11]. Nevertheless, it describes a variety of other problems as, e.g., a single impurity immersed in a Bose gas [35,36] or a molecular, localized defect in a quantum harmonic chain [37]. Moreover, it reveals the essential differences between a local interaction and potential quench [38]. The system remains stable for $K_{0} g l q_{c}>-\pi \mathrm{v}_{F}$; otherwise, one of the frequencies becomes imaginary, signaling an instability. Using the mapping [30] between quadratic bosonic models and a system of coupled harmonic oscillators, the confining parabola of the oscillators flattens and becomes inverted upon decreasing $g$ further. The short time decay features the universal time dependence as $\ln \mathcal{L}(t) \approx-t^{2} \operatorname{Tr}\left(G^{2}\right) \sim-\left(g K_{0} q_{c}^{2} t l\right)^{2}$, and the prefactor of the $t^{2}$ term is the variance of energy after the quench, as

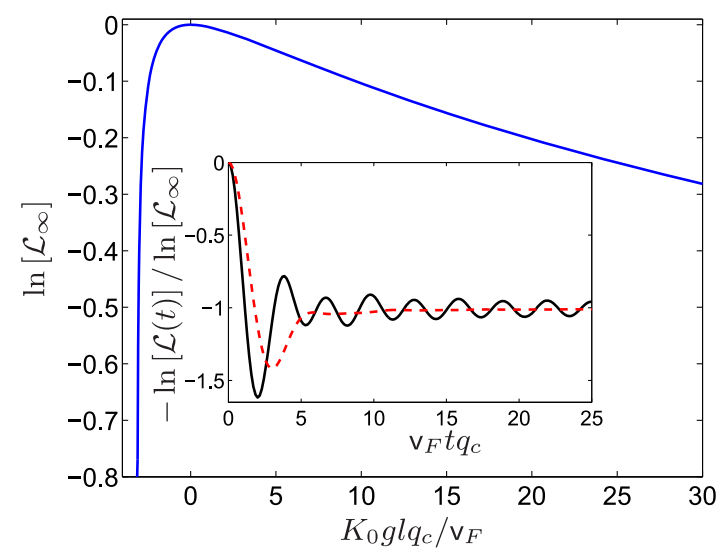

FIG. 2 (color online). The long time limit of the LE after a local interactions quench for $L q_{c}=4000$ is evaluated for the Luttinger model from Eq. (10). The inset depicts the time evolution for $K_{0} g l q_{c} / \mathrm{v}_{F}=-1$ and 2 from Eq. (12).

expected on general grounds [12]. In the long time limit (taking $L \rightarrow \infty$ first), the overlap tends to a finite, nonzero value (see Fig. 2), in sharp contrast to the case of a potential quench, signaling the absence of $\mathrm{OC}$ for the Luttinger model in the case of a local interaction quench.

Quenching over a finite spatial region $\left(q_{c} l \gg 1\right)$. - In this limit, the LE picks up distinct contributions from small and large momentum states. Because of the structure of the envelope function $\tilde{f}(k, l)$, a given momentum state $p$ can only be scattered within a $p \pm \pi / l$ momentum window, which becomes rather narrow with increasing $l$. In the $q_{c} l \gg 1$ limit, for large momentum states with $|p| \gg \pi / l$, the momentum remains an almost conserved quantum number, as its uncertainty $\pi / l$ is much smaller than its typical value $p$. Averaging over states within the narrow momentum shell $\pm \pi / l$, momentum conservation is regained in the $|p| \gg \pi / l$ region at the expense of enlarging the phase volume from $2 \pi / L$ to $2 \pi / l$. Therefore, the corresponding $\mathrm{LE}$ after an inhomogeneous quench is identical to that after a global quench [31], after replacing the minimum phase space volume $2 \pi / L$ by $2 \pi / l$ [40], regardless of the explicit value of $t$. In addition, the small momentum states with $|p| \ll \pi / l$ are spatially extended compared to $l$, therefore experiencing strong momentum scattering as if a local interaction quench has occurred, and the results of the previous section apply for these modes after replacing the upper momentum cutoff $q_{c}$ with $\pi / l$, yielding an $l$ independent dimensionless interaction strength as $K_{0} g l q_{c} / \mathrm{v}_{F} \rightarrow K_{0} g \pi / \mathrm{v}_{F}$. Since $l$ appeared only in this combination for a local quench, the contribution from states $|p| \ll \pi / l$ to the LE is independent of $l$. Consequently, the LE of an inhomogeneous quench, to leading order in $l$, is

$$
\frac{\ln \mathcal{L}(t)}{l}=-\int_{0}^{q_{c}} \frac{d q}{2 \pi} \ln \left[1+\frac{\sin ^{2}(\omega(q) t)}{4}\left(\frac{K}{K_{0}}-\frac{K_{0}}{K}\right)^{2}\right],
$$

with $\omega(q)=\mathrm{v}|q|$. 
In the long time limit with a $t \gg 1 / \mathrm{v} q_{c}$ limit, this gives

$$
\mathcal{L}_{\infty}\left(t \gg 1 / \mathrm{v} q_{c}\right)=\exp \left(-\gamma_{L L} \frac{l q_{c}}{\pi}\right),
$$

with

$$
\gamma_{\mathrm{LL}}=\ln \left[\frac{1}{2}+\frac{1}{4}\left(\frac{K}{K_{0}}+\frac{K_{0}}{K}\right)\right],
$$

which is related to the quantum geometric tensor of the $\mathrm{SU}(1,1)$ Lie group [41].

The short time decay, calculated from Eq. (10), features again the universal time dependence in the $t \ll 1 / \mathrm{v} q_{c}$ limit as $\ln \mathcal{L}(t) \approx-t^{2} \operatorname{Tr}\left(G^{2}\right)=-c\left(g K_{0} q_{c} t\right)^{2} q_{c} l$, with $c$ a nonuniversal constant. Using $g K_{0}=\mathrm{v}\left(K_{0} / K-K / K_{0}\right)$, this agrees with the short time expansion of Eq. (13).

For intermediate times, the LE oscillates around its steady state value with a frequency increasing with $g$, and the oscillations are under- or overdamped for $g \gtrless 0$, as shown in Fig. 3. The sharp oscillations arise mostly from the sharp cutoff scheme; a smoother (e.g., exponential) cutoff smoothens the oscillations. These are similar to the collapse and revival phenomenon of the LE in finite systems [12,42], although some of the excitation, created during the inhomogeneous quench, can be transmitted to the unquenched region, which then propagates freely and does not contribute to revivals any more as these do not interfere with other excitations. The steady state value of the LE agrees nicely with our analytical prediction in Eq. (14). For small $l$, slight deviations are visible with increasing $K\left(K_{0} g \rightarrow-\mathrm{v}_{F}\right)$ due to the contribution from small momentum states mostly, which experience a local interaction quench.

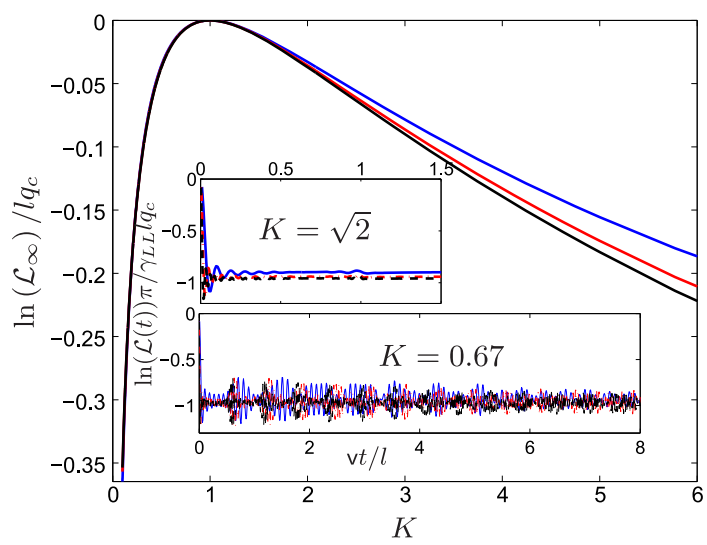

FIG. 3 (color online). The long time limit of the LE for the Luttinger model with $L q_{c}=2000$ is shown for $l q_{c}=40$ (blue curve), 120 (red curve), and 500 (black curve) evaluated from Eq. (12). The latter is indistinguishable from that after a homogeneous quench. The insets show the time dependence of the LE for the Luttinger model for various $K$ 's and $l q_{c}=40$ (blue solid line), 80 (red dash-dotted line), and 120 (black dashed line) from Eq. (10).
Luttinger model versus XXZ Heisenberg chain.-The Luttinger model description of the $X X Z$ Heisenberg chain neglects all sorts of additional terms [11,25] (e.g., band curvature or interaction), which are always present in lattice models. To establish the reliability of our bosonized calculations, we have evaluated the LE after an inhomogeneous quench using tDMRG methods [19-22] for the $X X Z$ Heisenberg chain, and the resulting data are plotted in Fig. 4 for system size $L=300$ and bond dimension 1000, chosen such that finite size and truncation effects are negligible. The simulations confirm the scaling of the echo with the size of the region $l$ as predicted in Eq. (14). Moreover, even the prefactor of the exponent can be estimated from the fidelity susceptibility $\chi_{f}$ around the $X X$ point of the Heisenberg model, leading to $q_{c} / \pi \approx$ $2 \chi_{f} \pi^{2}$, where $N$ is the number of lattice sites and $\chi_{f} \approx$ 0.0195 [43]. This simple estimate describes quantitatively the numerical data [44], in spite of the boundary backscattering terms in the inhomogeneous $X X Z$ chain [18], arising from the velocity mismatch between the noninteracting and interacting regions, regardless of the sign of $J_{z}$ [11], as shown in the inset of Fig. 4.

An interaction quench over a spatially finite region is realizable experimentally, using a hybrid setup containing a cold atomic LL [45] and a flux qubit proposed in Ref. [31] to measure the LE after a homogeneous quench. In addition to an external magnetic field, which tunes the properties of the qubit, the induced magnetic field of the flux qubit itself controls the interaction strength in the LL via a Feshbach resonance. As we have shown here, by quenching the interaction over a finite spatial region $1 / q_{c} \ll l \ll L$ (and not homogeneously over the whole system), the observation

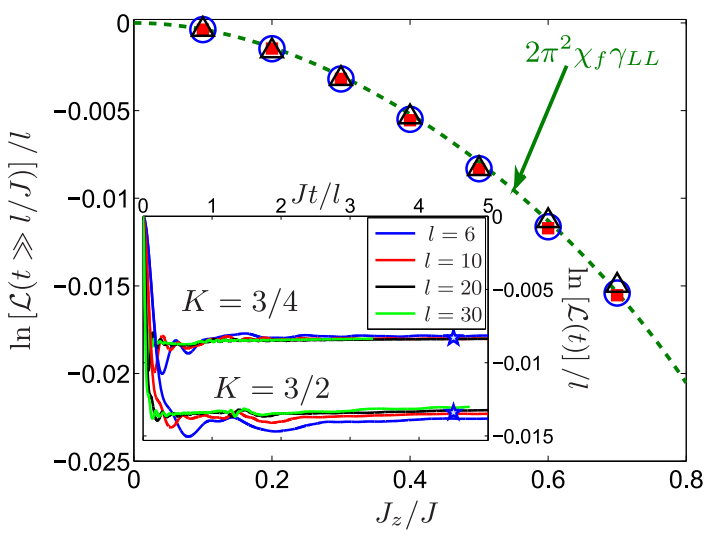

FIG. 4 (color online). The inhomogeneous Loschmidt echo of the $X X Z$ chain for $L=300$ from tDMRG, starting from the $X X$ point to several final $J_{z} / J$ for $l=10$ (blue circles), 40 (red squares), and 120 (black triangles), and the green dashed line is Eq. (14). The inset shows the time evolution of the LE for $J_{z}=$ $0.5 J(K=3 / 4)$ and $-0.5 J(K=3 / 2)$ for several $l$ 's, as indicated by the legend. The pentagons indicate the long time asymptotes of $\ln [\mathcal{L}(t \rightarrow \infty)] / L$ after a homogeneous quench from Ref. [31] in the $L \rightarrow \infty$ limit. 
of the peculiar scaling of the overlap as in Eq. (14) in terms of the Luttinger liquid parameters is reachable.

To conclude, we have demonstrated that no orthogonality catastrophe occurs during the time evolution of the Loschmidt echo in Luttinger liquids following an interaction quench within a finite spatial region-in sharp contrast to a potential quench. The comparison of the bosonization results to numerical simulations of the $X X Z$ Heisenberg chain, which contains local backscatterers at the boundary of the quenched region, demonstrates the applicability of the Luttinger liquid concept.

This research has been supported by the Hungarian Scientific Research Funds No. K101244, No. K105149, and No. K108676, by the ERC Grant No. ERC-259374Sylo, and by the Bolyai Program of the HAS.

*dora@eik.bme.hu

[1] T. Kinoshita, T. Wenger, and D. S. Weiss, Nature (London) 440, 900 (2006).

[2] A. Polkovnikov, K. Sengupta, A. Silva, and M. Vengalattore, Rev. Mod. Phys. 83, 863 (2011).

[3] J. Dziarmaga, Adv. Phys. 59, 1063 (2010).

[4] M. Campisi, P. Hänggi, and P. Talkner, Rev. Mod. Phys. 83, 771 (2011).

[5] T. Oka and H. Aoki, Quantum and Semi-classical Percolation and Breakdown in Disordered Solids, Lecture Notes in Physics Vol. 762 (Springer, Berlin, 2009).

[6] I. Bloch, J. Dalibard, and W. Zwerger, Rev. Mod. Phys. 80, 885 (2008).

[7] S. Sotiriadis and J. Cardy, J. Stat. Mech. (2008) P11003.

[8] M. S. Foster, E. A. Yuzbashyan, and B. L. Altshuler, Phys. Rev. Lett. 105, 135701 (2010).

[9] J. Lancaster and A. Mitra, Phys. Rev. E 81, 061134 (2010).

[10] J. Dziarmaga and M. M. Rams, New J. Phys. 12, 055007 (2010).

[11] A. O. Gogolin, A. A. Nersesyan, and A. M. Tsvelik, Bosonization and Strongly Correlated Systems (Cambridge University Press, Cambridge, England, 1998).

[12] A. Peres, Phys. Rev. A 30, 1610 (1984).

[13] I. Affleck and A. W. W. Ludwig, J. Phys. A 27, 5375 (1994).

[14] M. Schiró and A. Mitra, Phys. Rev. Lett. 112, 246401 (2014).

[15] P. Smacchia and A. Silva, Phys. Rev. Lett. 109, 037202 (2012).

[16] A. Gambassi and A. Silva, Phys. Rev. Lett. 109, 250602 (2012).

[17] D. Rossini, T. Calarco, V. Giovannetti, S. Montangero, and R. Fazio, Phys. Rev. A 75, 032333 (2007).

[18] N. Sedlmayr, J. Ohst, I. Affleck, J. Sirker, and S. Eggert, Phys. Rev. B 86, 121302 (2012).
[19] G. Vidal, Phys. Rev. Lett. 93, 040502 (2004).

[20] A. J. Daley, C. Kollath, U. Schollwöck, and G. Vidal, J. Stat. Mech. (2004) P04005.

[21] A. E. Feiguin and S. R. White, Phys. Rev. B 72, 020404 (2005).

[22] U. Schollwöck, Ann. Phys. (Amsterdam) 326, 96 (2011).

[23] L. M. Robledo, Phys. Rev. C 79, 021302 (2009).

[24] N. Onishi and T. Horibata, Prog. Theor. Phys. 64, 1650 (1980).

[25] T. Giamarchi, Quantum Physics in One Dimension (Oxford University Press, Oxford, England, 2004).

[26] I. Safi and H. J. Schulz, Phys. Rev. B 52, R17040 (1995).

[27] D. L. Maslov and M. Stone, Phys. Rev. B 52, R5539 (1995).

[28] V. V. Ponomarenko, Phys. Rev. B 52, R8666 (1995).

[29] R. Thomale and A. Seidel, Phys. Rev. B 83, 115330 (2011).

[30] G. D. Mahan, Many Particle Physics (Plenum, New York, 1990).

[31] B. Dóra, F. Pollmann, J. Fortágh, and G. Zaránd, Phys. Rev. Lett. 111, 046402 (2013).

[32] R. Sachdeva, T. Nag, A. Agarwal, and A. Dutta, Phys. Rev. B 90, 045421 (2014).

[33] F. A. Berezin, The Method of Second Quantization (Academic Press, New York, 1966).

[34] M. Tegmark and L. Yeh, Physica (Amsterdam) 202A, 342 (1994).

[35] C. Zipkes, S. Palzer, C. Sias, and M. Köhl, Nature (London) 464, 388 (2010).

[36] C. Gaul and C. A. Müller, Phys. Rev. A 83, 063629 (2011).

[37] S. Brühl, E. Sigmund, and M. Wagner, Ann. Phys. (Berlin) 489, 219 (1977).

[38] Equation (5) can be evaluated using a cumulant expansion. For a local potential quench, it amounts to calculating a Cauchy determinant [39], i.e., the determinant of a matrix with elements $a_{i, j} \sim 1 /(i-j)$. For a local interaction quench, the evaluation of a generalized Cauchy determinant is required with elements $a_{i, j} \sim 1 /(i-j)^{2}$, for which no useful results are available. We rely on the inhomogeneous Bogoliubov equations and Eq. (10) instead.

[39] G. Yuval and P. W. Anderson, Phys. Rev. B 1, 1522 (1970).

[40] Changing the minimal momentum from 0 to $\pi / l$ gives an $O(1)$ correction; thus, it is negligible.

[41] J. P. Provost and G. Vallee, Commun. Math. Phys. 76, 289 (1980).

[42] L. Campos Venuti and P. Zanardi, Phys. Rev. A 81, 022113 (2010).

[43] J. Sirker, Phys. Rev. Lett. 105, 117203 (2010).

[44] Though we have mostly considered even l's on the $X X Z$ chain, let us note that odd l's behave slightly differently: the LE decays faster in time before saturation, though the evenodd difference diminishes with increasing $l$.

[45] M. A. Cazalilla, R. Citro, T. Giamarchi, E. Orignac, and M. Rigol, Rev. Mod. Phys. 83, 1405 (2011). 